\title{
The Utility of APEX in the Context of Database Education
}

\author{
Hyontai Sug1)
}

\begin{abstract}
Developing database applications usually takes two-tier approach[1]. The first tier is related to developing databases and related database programming, and the second tier is related to developing graphical user interface(GUI). As a result, developing database applications requires comprehensive skills in computer engineering, and assigns developers a lot of work to do, and the workloads may become very heavy, especially if the developers are students. But, nowadays, because of the popularity of good GUI in personal IT devices like smartphones, users of IT systems expect better GUI for their applications[2]. Most curriculum in computer engineering has database programming course, but it's hard to cover both subjects in the course because of the complexity of database language, SQL, as well as the design and related theory of databases. In this sense rapid database application building tools that have capability of building GUI easily may help developers a lot, because they can allow developers to focus on designing and developing database part more. In this paper, the functionalities of one of rapid database application tools called APEX is investigated for such purpose, and utility of it is explained especially in the context of database education.
\end{abstract}

Keywords: Graphical User Interface, Rapid, Database, Application, Development, SQL, Education

\section{Introduction}

The Information age is characterized by the rapid shift from traditional industry to an economy primarily based on information technology. While the information technology contains a lot of hardware and software systems, one central part of it is database technology, because each component of related systems may generate data and the data need to be stored. As a result, ACM(Association for Computing Machinery) and IEEE computer society considered that database courses is very important for the education of computer science, and recommended database curriculum[3]. Based on internet search for the curriculum of department of computer engineering in Korea, among fifteen computer-related departments, one department has three database courses, five other departments have one database course, and nine another departments have two database courses, one for theory, and the other for practice or database programming as of August 2019. As for database programming course, SQL-based curriculum is

Received(September 18, 2019), Review Result(1st: October 7, 2019, 2nd: November 18, 2019), Accepted(January 30, 2020)

1) (Professor) 47011 Div. Computer Engineering, Dongseo Univ., 47 Jurye-ro, Sasang-gu, Busan, Korea email: sht@dongseo.ac.kr 
very common, because SQL which stands for Structured Query Language is the standard language for relational databases, and most operational databases in the world are based on relational databases[4].

SQL is very large language, and becomes more complex as new versions are adapted. The first standardization of SQL is called SQL-86, and the latest is called SQL:2019. For example, ISO SQL 2011 Standard document ISO/IEC 9075-1:2011 Information technology -- Database languages -- SQL -- Part 1: Framework (SQL/Framework) has 90 pages, and the document consists of 14 parts[5]. As a result, most database programming courses may cover SQL part only. But, nowadays, people are very concerned with GUI in their applications due to wide availability of smart devices. But, it's not easy to cover the both subjects of database and GUI programming in one database course in full depth. For example, HTML5 and javascript that are teaching subjects of web programming to build web-based GUI may occupy one course, and most computer departments have such curriculum. As a result, a database programming course may not be a satisfactory course for learners, because the both subjects are difficult to be covered in one course. In this sense, rapid database application development tools that have easy graphical ability may satisfy the learners as well as the educators for a database course. In section 2, we'll see how we can develop such graphical database applications easily using APEX, and in section 3 some conclusions will be presented.

\section{Database Application Development Using APEX}

\subsection{APEX}

APEX stands for APplication EXpress. The name reflects that we may develop database applications that have web-based interfaces very rapidly or effortlessly. APEX was developed by Oracle, the leading database company[4], and comes with Oracle database management system(DBMS) starting from Oracle 11g. When we install Oracle DBMS version $11 \mathrm{~g}$ and $12 \mathrm{c}$, it is installed by default. Moreover, when we install Oracle $11 \mathrm{~g}$ or $12 \mathrm{c}$ express edition which is free, APEX is also installed by default. While Oracle DBMS is good for large databases, Oracle express edition is good for users whose database is not so big. In other words, Oracle express edition can store up to $11 \mathrm{~GB}$ of user data, and use up to $1 \mathrm{~GB}$ of memory and one CPU. APEX can be used to build web applications linked with underlying Oracle database without much or no web programming. So, database developers or students may use it to build web-based user interfaces of database applications easily. 
Building GUI for database applications usually needs lots of work to do. Usually, the tools to build GUI are independent from underlying DBMS. For example, when we build java-based applications, we may use SWING[6] or JSP[7]. Swing is the standard tool in JDK (Java Development Kit) to build GUI for java application programs. Web application servers that run JSP insert related java codes into HTML codes to make web pages, and transfer the codes to web browser in clients. The other popular language to build web applications in the server side is PHP[8]. PHP is a hypertext preprocessor. It is known that programming in PHP is relatively easier than in JSP because the language is made for such purpose. From this facts we can see that developing GUI for databases needs quite much work to do, because the two tools to build GUI and database are independent. But, users usually expect that database applications should have some good user interfaces. If we may use relatively simple tools to build database applications, such expectation may be fulfilled easily. For example, if we use Microsoft ACCESS that is often used to build small databases, we can build GUI easily. Users or developers of Microsoft ACCESS can build GUI called forms, and generate good looking reports easily for small databases[9]. The convenience in using graphical tools of Microsoft ACCESS make it a comprehensive tool for small database applications so that it can be a good tool for practice in teaching introductory course of information systems or databases. For example, MIT professor Dellarocas teaches Microsoft ACCESS for GUI building in their information technology course at the sloan school of management, MIT since 2003 as open course for graduate students[10]. In the course students are taught how to build forms that can be used to enter data, and how to generate reports that can be used to make reports of user's needs. But, such comprehensive and fast GUI-based application building tools are very rare for large databases. On the other hand, students of computer engineering need to learn how to use large databases. In this sense Oracle APEX is a good tool for such purpose, because educators may focus on teaching the technologies for large databases more and students who are familar with smart devices may build GUI for their capstone projects easily.

\subsection{How to Build Database Applications with APEX}

\subsubsection{Database Tables}

There are several ways to build database applications with APEX. A simple way to start is to use existing tables. Tables can be defined by SQL that can be covered by conventional database programming course[11]. If we install Oracle $11 \mathrm{~g}$ release 2 expression edition[12], we'll have 
system account as well as a practice account called HR. But, it is locked, so you have to unlock HR account from system account using a command like 'ALTER USER HR ACCOUNT UNLOCK;'. HR account has the following ready-made tables, where primary keys are underlined.

EMPLOYEES(EMPLOYEE_ID, FIRST_NAME， LAST_NAME， EMAIL， PHONE_NUMBER, HIRE_DATE, JOB_ID, SALARY, COMMISSION_PCT, MANAGER_ID, DEPARTMENT_ID)

DEPARTMENTS(DEPARTMENT_ID, DEPARTMENT_NAME, MANAGER_ID, LOCATION_ID) LOCATIONS(LOCATION_ID, STREET_ADDRESS, POSTAL_CODE, CITY, STATE_PROVINCE, COUNTRY_ID)

COUNTRIES(COUNTRY_ID, COUNTRY_NAME, REGION_ID)

REGIONS(REGION_ID, REGION_NAME)

JOBS(JOB_ID, JOB_TITLE, MIN_SALARY, MAX_SALARY)

JOB_HISTORY(EMPLOYEE_ID, START_DATE, END_DATE, JOB_ID, DEPARTMENT_ID)

There is one view called EMP_DATAILS_VIEW.

EMP_DETAILS_VIEW(EMPLOYEE_ID, JOB_ID, MANAGER_ID, LOCATION_ID, COUNTRY_ID, FIRST_NAME, LAST_NAME, SALARY, COMMISSION_PCT, DEPARTMENT_NAME, JOB_TITLE, CITY, STATE_PROVINCE, COUNTRY_NAME, REGION_NAME)

Because we already have tables, we can make automatic forms of each tables or views with APEX easily. Let's see how we can make them in the next section. The process is very simple.

\subsubsection{How to Use APEX}

The first step is to login APEX system. We can login the system by double-clicking XE icon as in [Fig. 1] in desktop interface. The icon is installed automatically when we install Oracle $11 \mathrm{~g}$ r2 express edition.

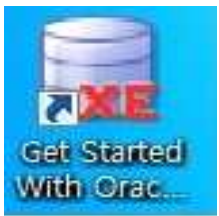

[Fig. 1] XE Icon

If we see a home page like in [Fig. 2] after double clicking, we are executing the first web 
page where we can choose APEX application express.

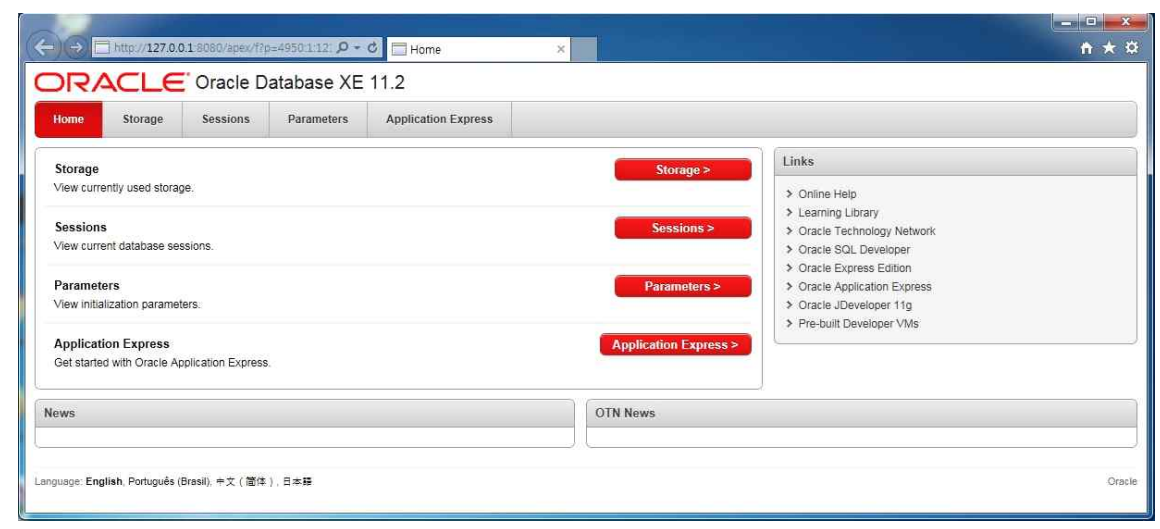

[Fig. 2] The First Web Page

When we click 'Application Express' Button in the web page, a login dialog box will be popped. So we should login as system. If we haven't created any work space, the next step is creating a work space first. We can make a work space and related account in the web page like [Fig. 3].

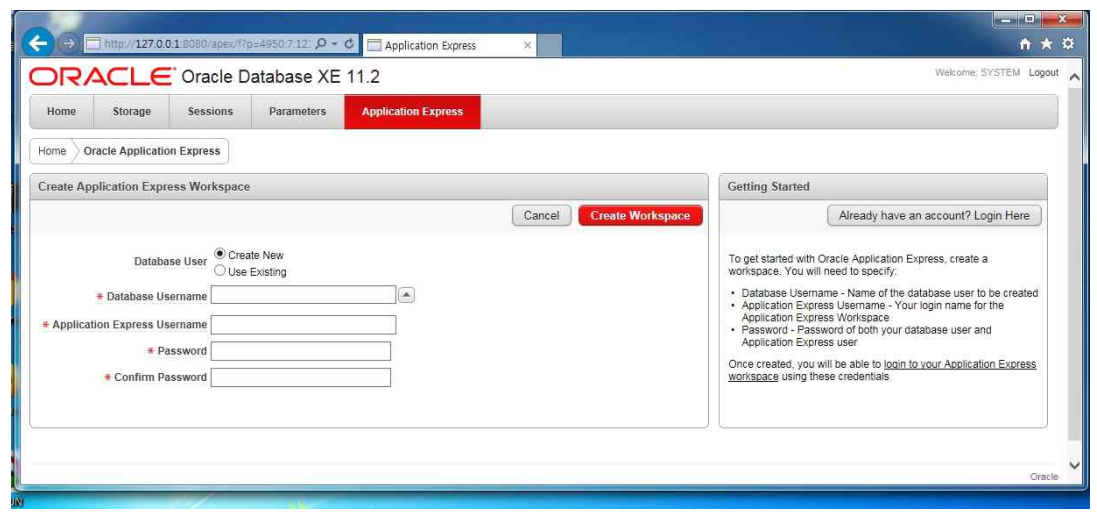

[Fig. 3] Creating Workspace and Database User

Because we have HR account that has tables for our example application, we may enter the same user name and password for database user name and application express user name to create our work space in [Fig. 3]. If we have created accounts already, we can choose 'Already have an account? Login Here' button that is located at right side of the web page. After creating the work space, we see the web page like in [Fig. 2] again. If we click 'Application 
Express' button again, a login dialog box will be popped. So, we can login the system by entering user name and password that have been created at the stage in [Fig. 3]. After logging the account, we can see main page of application express like in [Fig. 4].

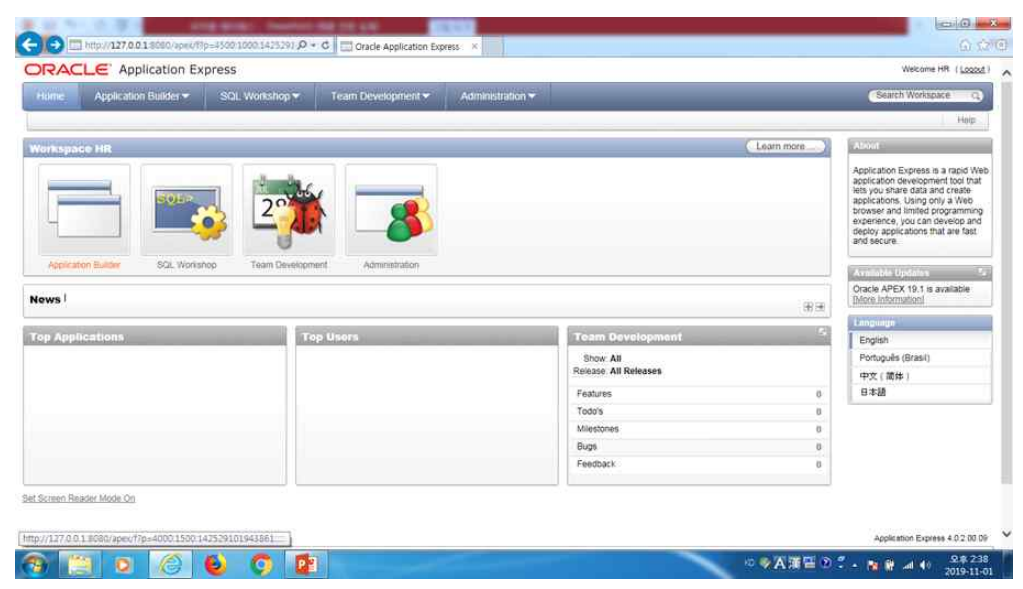

[Fig. 4] Main Page of Application Express

From this main page of application express, we can start to create our own applications. We can choose 'Application Builder' icon or menu to create our own applications. Because we already have tables, creating corresponding forms is straight forward.

After choosing 'Application Builder', we can choose menus in the following sequence, 'Create $>$ Database (as application type) >From Scratch'. Then, we can enter or use default values for application name and a unique application ID, after that we can select an application method and a schema. The schema in Oracle is user name, which is HR for our example. [Fig. 5] shows the corresponding dialog box where default values are used.

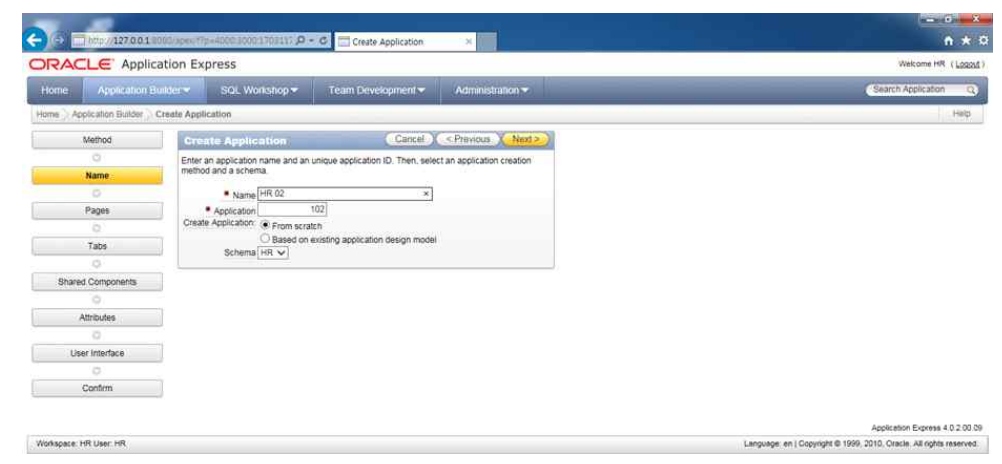

[Fig. 5] Entering Application Information 
Next step is to choose an application type. There are seven different application types 'Blank', 'Report', 'Form', 'Report and Form', 'Tabular Form', 'Master Detail', and 'Chart'. As our example, we choose 'Report and Form' that can display data in a table or view in tabular and form format, then we choose tables or views that we want to work. After confirmation, we can run the application. The following [Fig. 6] shows the result.

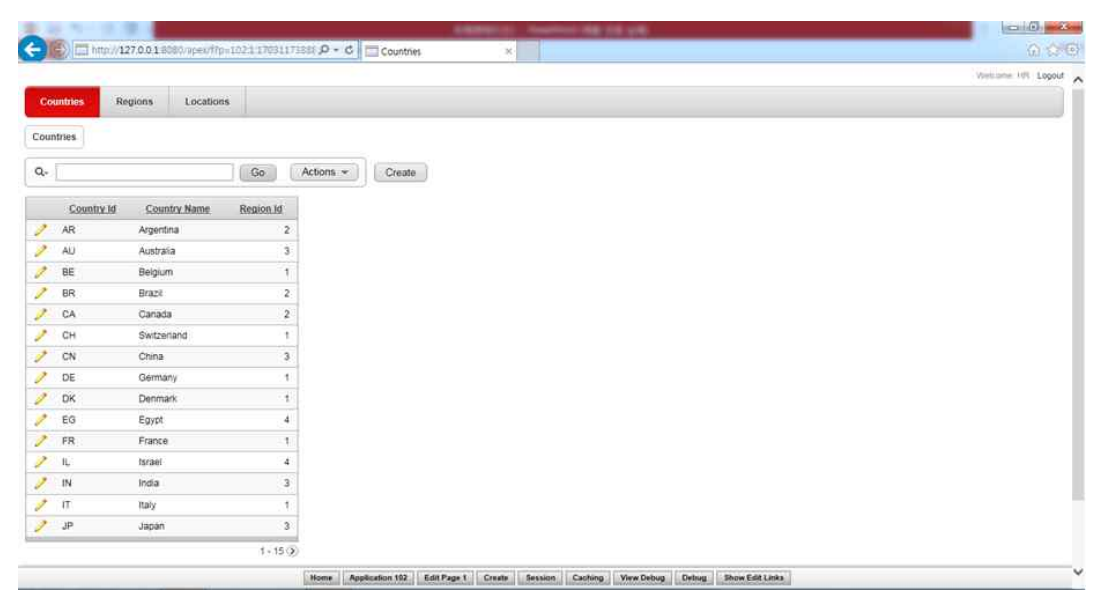

[Fig. 6] Web Pages of our Application

If we click the pencil icon at the left of each row, we can edit the corresponding record. [Fig. 7] shows the record in form format.

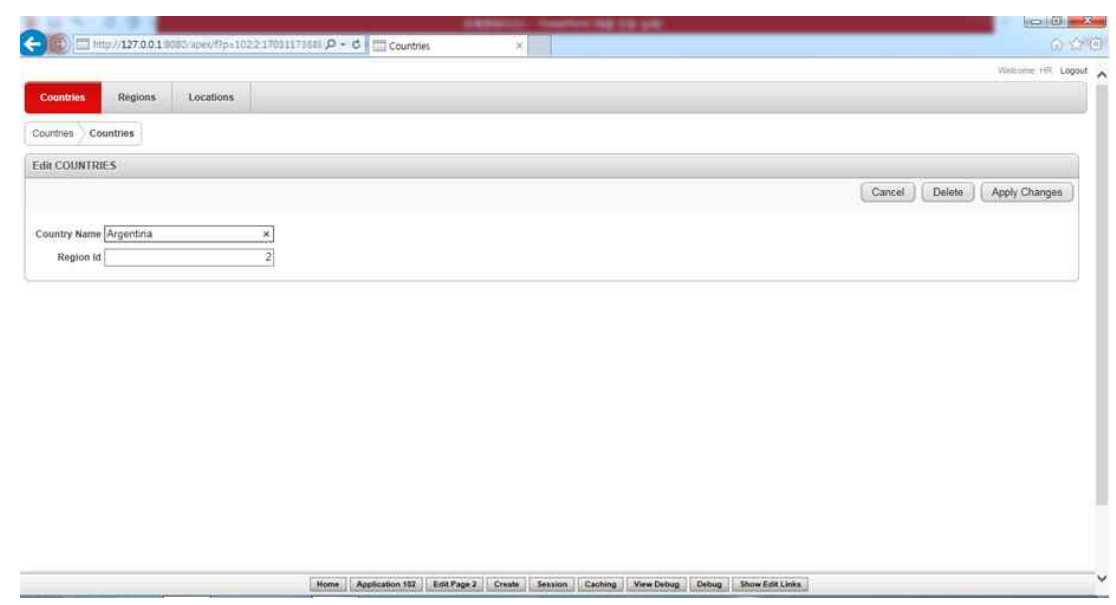

[Fig. 7] A Record in Form

We can also generate reports easily in existing applications. Instead of choosing 'Run 
Application', we can choose 'Create' button that can be found at the right middle of the application builder. We can choose 13 different type of applications like 'Blank Page', 'Multiple Blank Pages', 'Report', 'Chart', 'Form', 'Wizard', 'Calendar', 'Tree', 'Login Page', 'Access Control', 'Page Zero', 'Map', and 'Feedback Page'. For our example, we choose 'Report'. After that we choose 'Classic Report' among 4 different report types, 'Interactive Report', 'Classic Report', 'Report on Web Service Result', and 'Wizard Report'. Then we may enter appropriate information for classic report. [Fig. 8] shows the dialog box which shows default values.
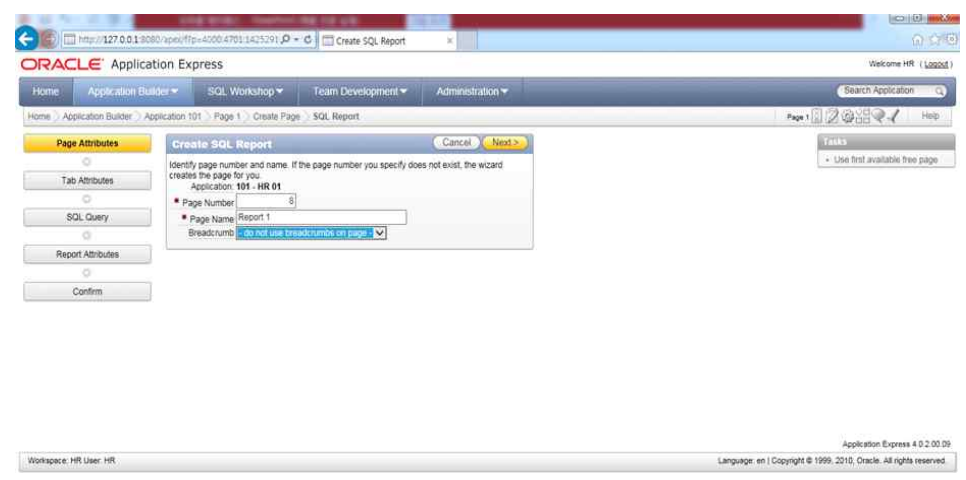

[Fig. 8] Creating SQL Report

The next dialog box is for using 'tab' for the report. We may choose default value that is not using tab in the report. Then we may enter an appropriate SQL statement for our report. [Fig. 9] shows a SQL statement, 'SELECT * FROM EMPLOYEES;' for our report for the simplicity of example. The SQL statement outputs all the information of employees stored in 'EMPLOYEES' table.

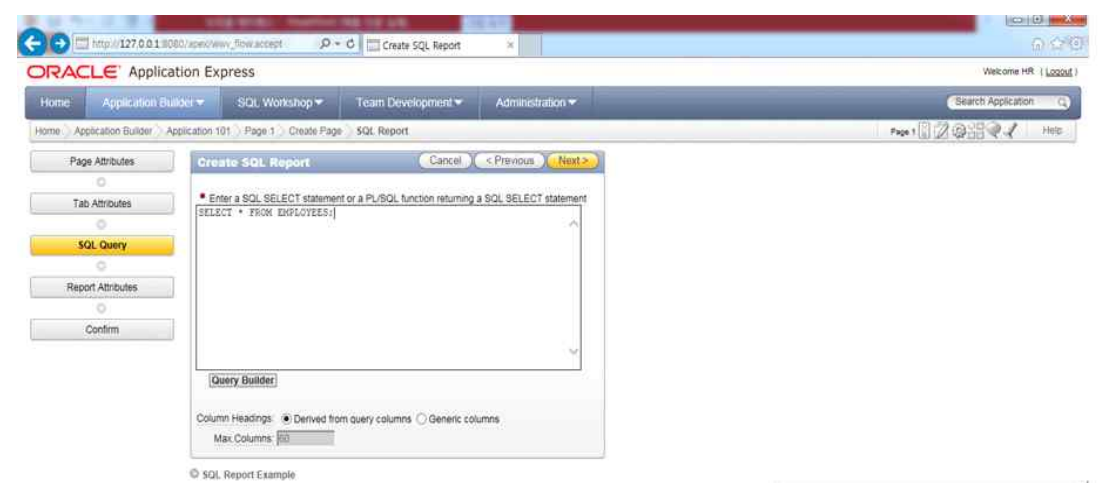

[Fig. 9] Entering SQL Statement for the Report 
After confirmation steps of 2 phases, we can run our report. [Fig. 10] shows the generated report.

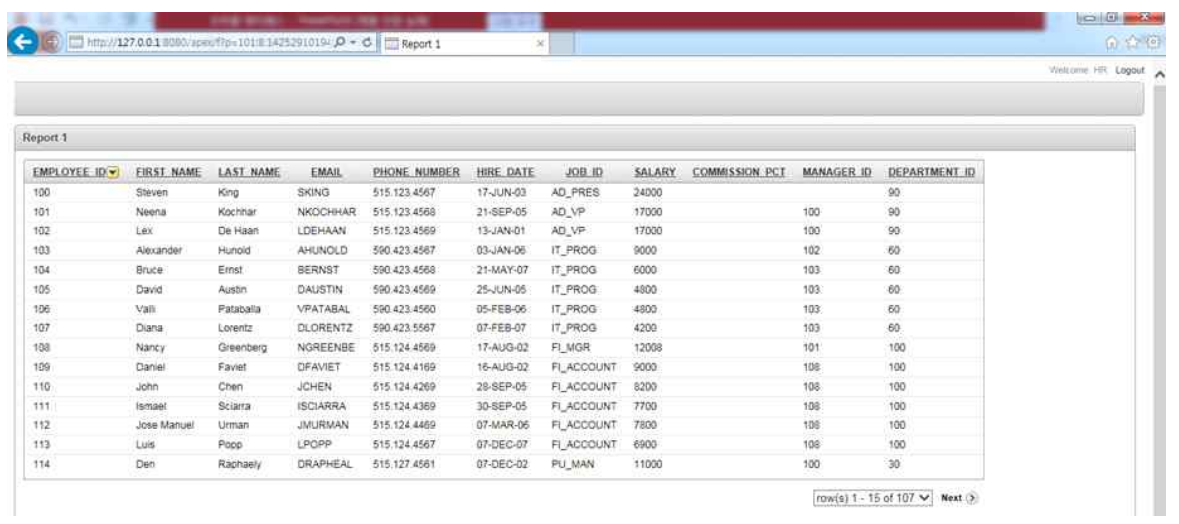

[Fig. 10] The Generated Report

\section{Conclusions}

Developing database applications needs comprehensive skills in computer engineering, and demands a lot of work to do. When we develop database applications in conventional way, we need to create databases, and we also need to develop GUI and application logic for the database applications. And this approach is considered as a good way to develop database applications. But, here, one more thing to consider when we teach students database programming especially related to GUI. Because we are living in smart age, students may expect that their capstone projects of database course have good user interfaces, but, the expectation needs lots of work to do, if the students should follow in conventional way.

Most curriculum in computer engineering or computer science has database programming courses, but it's hard to cover the both subjects of GUI and database programming in one course because of the complexity of database language, SQL, as well as design and related theory of databases, and also GUI programming like web programming. In this sense rapid database application development tools may help educators a lot, because they can focus on database part more for more effective database education. Moreover, such tools may satisfy students also, because they can get good GUI for their applications. In this paper, the functionalities of one of rapid database application building tools called APEX is investigated for that purpose, and its utility is explained, and shown how easy to use it, if we know SQL which is taught by conventional database courses. As a conclusion, we may say that it is 
worthwhile to adept APEX as a part of database education. Additional good point of APEX is that it is installed automatically when we install Oracle $11 \mathrm{~g}$ or $12 \mathrm{c}$ express edition that is freely available, so that no additional burden for the work.

\section{References}

[1] M.E. Patil, R.N. Mulchndani, R.R. Ahuja, Design and Implementation of Graphical User Interface for Relational Database Management System, International Journal of Computer Science and Information Technologies, (2012), Vol.3, No.3, pp.3871-3874.

[2] G. Ines, S. Makram, C. Mabrouka, A. Mourad, Evaluation of Mobile Interfaces as Optimization Problem, International conference on Knowledge Based and Intelligent Information and Engineering Systems, KES2017, 6-8 September 2017, Marseille, France, Procedia Computer Science, Vol.112, (2017), pp.235-248. https://doi.org/10.1016/j.procs.2017.08.234

[3] https://www.acm.org/binaries/content/assets/education/cs2013_web_final.pdf, Dec 20 (2013)

[4] https://www.statista.com/statistics/809750/worldwide-popularity-ranking-database-management-systems, Sep 3 (2019)

[5] https://www.iso.org/standard/53681.html, Dec 1 (2011)

[6] H. Yang, Java Swing Tutorials - Herong's Tutorial Examples, Independently published, (2018)

[7] J. Murach and M. Urban, Murach's Java Servlets and JSP, 3rd ed., Mike Murach \& Associates, Inc. (2014)

[8] R. Nixon, Learning PHP, MySQL \& JavaScripts: With jQuery, CSS \& HTML5, 5th ed., O'reilly Media, (2018)

[9] M. Alexander and R. Kusleika, ACCESS 2019 Bible, Wiley, (2018)

[10] https://ocw.mit.edu/courses/sloan-school-of-management/15-564-information-technology-i-spring-2003/lecture-notes, Jan 2 (2003)

[11] R. Elmasri, S.B. Navathe, Fundamentals of database Systems, 7th ed., Pearson, (2016)

[12] https://www.oracle.com/technetwork/es/database/express-edition/overview/index.html, Dec 1 (2019) 